cases of damage of the internal epicondyle or in the condylar groove, and the nerve should be exposed only if symptoms persist. It may be necessary to transpose it anteriorly.

Late ulnar palsy not infrequently follows fracture and displacement of the external condyle. The fracture dates nearly always from early childhood, and the neuritis occurs many years later. It is a traction lesion predetermined by a cubitus valgus deformity. The nerve trunk, being destined to take a longer course, is stretched in the process. It will be necessary to transpose the course of the nerve from its groove to the front of the joint, and then recovery may be expected.

The median nerve is occasionally injured in supracondylar fractures by the sharp projecting angle of the lower end of the diaphysis, and this may occur as an immediate complication of the fracture, or may follow a rough attempt at reduction. Direct injury to the musculo-spiral nerve is rare, but when it occurs the prognosis after operation is good.

\section{Myositis Ossificans}

This alarming complication may arise after any fracture about the elbow, but is most commonly associated with dislocation of the elbow backwards. Its onset is gradual, and, when established, may result in a complete or partial anklylosis of the elbow-joint. It is generally due to tearing of the muscular and periosteal tissue from bone accompanied by haemorrhage. Fragments of periosteum and osteogenetic tissue are pulled away, and probably in this way the formation of new bone takes place along the interfibrillary and intermuscular septa. The symptoms are insidious. An elbow which is doing well begins to get stiff in the third or fourth week, and, omitting to take an $x$-ray film, a practitioner prescribes passive movements. This is a dangerous procedure. The film shows a suspicious cloudiness about the attachments of some muscle-usually the brachialis anticus. In two or three weeks this shadow becomes more dense, and traces of bone structure are noted. It is a mistake at this stage to operate, because further osteogenesis often occurs. The elbow should be kept quite still until evidence is obtained that bone deposit has ended - a fact which is easily ascertained by successive radiograms-and then only should active movements be allowed. In rare instances the bone may be completely absorbed, but in many cases partial absorption occurs. If the deposit remains and is inactive, but causes disability, it can now be removed, but if the movements of the elbow are free it should be ignored. These deposits may remain for many months, sometimes years, and ultimately be absorbed.

While, therefore, we have every right to expect a good result from injuries of the elbow, we should be alive to possibilities and dangers. We should carefully note any nerve involvement at the moment of injury; we should speak of the possibilities of myositis ossificans, and warn the parents of the dangers of energetic massage and movement campaigns. We should also recollect that the practitioner is not necessarily to blame if an ischaemic palsy occurs it may have nothing to do with tight bandaging, and it has occurred without flexion of the elbow, without splints, and without bandaging. At the same time we should, if we can, prevent pressure from within by reducing displacements which cause acute venous obstruction, and avoid pressure from without. By no argument can the practitioner be held guilty of the origin of a myositis ossificans, and neither can he be blamed for its development, provided that he does not prescribe passive movements during the period of bone formation. If myositis ossificans appears and any error is to be made let it be on the side of rest; I have seen many tragedies occur from too early movement. Finally, do not fully flex an elbow without first reducing the fracture or displacement, and, having flexed the elbow, do not obstruct the circulation of the arm by bandaging it in the flexed position.

\section{REFERENCES}

1. Jones, Sir Robert: Volkmann's Ischaemic Contracture, with Special Reference to Treatment, British Medical Journal, 1928, ii, 639.

${ }^{2}$ An Anatomical and Surgical Study of Fractures of the Lower End of the Humerus. The Samuel Gross Prize Essay. Lea and Febiger, New York.

\section{THE TREATMENT OF TETANUS}

LESLIE COLE, M.A., M.D.CANTAB., M.R.C.P.

HONORARY PHYSICIAN TO ADDENBROOKE'S HOSPITAL, CAMBRIDGE : UNIVERSITY TEACHER IN MEDICINE; CONSULTING PHYSICIAN TO HUNTINGDON COUNTY HOSPITAL

Tetanus is now a comparatively rare disease in civil practice, and this is largely due to the prophylactic use of antitoxin. In agricultural districts such as East Anglia, however, it still occurs too frequently, often after wounds which are not seen by a doctor, or which seem so trivial that antitoxin is not given. Ten cases have been admitted and treated at Addenbrooke's Hospital during the last three years, and five of these have recovered. These are recorded to emphasize the danger of tetanus that still exists, and for any light that they may throw on the best methods of treating the disease.

\section{CASE I}

Male, aged 11 years. Fourteen days before admission a sharp splinter of wood entered the skin below the left knee and penetrated $1 / 2$ inch to the bone. This was removed by a doctor, and the wound healed normally. Three days before admission he complained that the left leg was rather stiff. The stiffness spread in a $f \in w$ hours to the right leg, and he found that he could not open his mouth.

State on Admission.-Risus sardonicus ; rigidity of the neck and spine; board-like abdomen. The teeth could only be separated a distance of $1 / 4$ inch, but he could speak and could swallow fluids. Severe spasms occurred with opisthotonos about every half-hour. The only sign of a wound was a small healed scar, and this was not touched.

Treatment and Progress.-On the day of admission 70,000 units of antitoxin were given intrathecally, 60,000 intramuscularly, and 30,000 intravenously. (International units are used throughout.) This was done under chloroform anaesthesia. Bromide and chloral were also given. The next day he was definitely better, and from this time steadily improved, the spasms gradually becoming less frequent and less severe. In the following six days 74,000 units in all were given, the dosage being gradually reduced: 44,000 were given intrathecally and 30,000 intramuscularly. At the end of one week he was well but for slight stiffness of the jaw and of the abdominal muscles, which had quite disappeared in fourteen days.

\section{CASE II}

Male, aged 23 years, horsekeeper. No history of injury or illness. Quite well and at work until fourteen days before admission. He then complained of stiffness of the back. Two days later his jaw also became stiff. These symptoms continued, but he did not send for the doctor until he had had them eight days. The stiffness gradually increased, and spasms of pain in the back and chest and neck came on after eight days. He used to get out of bed and walk round the room in an attempt to work off the stiffness.

State on Admission.-All the muscles of the chest, neck, and abdomen were in a state of severe spasm, which was made worse by any stimulus, such as movement. Boardlike rigidity of the abdomen was present; risus sardonicus marked. The teeth could not be separated more than $1 / 4$ inch. Sweating was profuse.

Treatment and Progress.-On the day of admission 40,000 units were given intrathecally, and the same amount intravenously under chloroform anaesthesia. A 
further 80,000 intrathecally, 60,000 intravenously, and 20,000 intramuscularly were given during the next six days. The spasms had then stopped, stiffiess was very much less, and the teeth could be $\mathrm{s}$ parated a distance of $1 \frac{1}{2}$ inches. The patient was discharged well after sixteen days. No sign of a wound or scar could be found.

\section{CASE III}

A Male, aged 10 years. Fourteen days before admission his father noticed that he was limping. Nine days later he seemed unwell, and complained of pain and stiffness in the back and neck. This was followed by increasing stiffness of the jaw, so that on the day of admission he was unable to open his mouth.

State on Admission.-An extremely dirty child. Small abrasion on the right foot, with scab formation. Marked stiffness of all the muscles of the back, chest, and neck. Board-like rigidity of the abdomen. Risus sardonicus marked. Teeth could be separated a distance of $1 / 4$ inch between spasms. Occasional spasms followed movement or irritation.

Treatment and Progress.-The scab was removed and the wound dressed with hydrogen peroxide dressings. On admission 40,000 units were given intrathecally and the same amount intravenously. These amounts were repeated intravenously and intramuscularly on the following day. During the next ten days 120,000 units were given intravenously, 40,000 intrathecally, and 20,000 intramuscularly. Five days after ad rission the spasms had ceased and he was very much better. At the end of three weeks he could sit up in bed and feed himself. He was discharged cured in five weeks.

\section{CASE IV}

Male, aged 11. Twenty-one days before admission a thorn entered the right leg just below the knee. The leg was bathed, and boric lint was applied and worn for several days until the wound appeared to have healed up satisfactorily. The patient remained quite well for sixteen days, when he complained that he felt queer. On the following day he could not eat, and bit his tongue. Two days before admission he could not open his mouth, and on the day of admission spasms appeared.

State on Admission.--Severe stiffness of the muscles of the neck and chest; board-like rigidity of abdomen. Risus sardonicus present. The teeth could not be separated more than $1 / 4$ inch between spasms. Spasms of the whole body were very severe, and occurred with opisthotonos every five minutes, or on the slightest stimulation. There was a small infected wound with a scab below the right knee.

Treatment and Progress.-The wound was opened up and a cavity was found extending down to the bone, containing 1 c.cm. of foul pus and a thorn. This was thoroughly opened up and irrigated with hydrogen peroxide, and a drain was inserted : 80,000 units were given intravenously, 40,000 intramuscularly, and 40,000 in the region of the wound. An injection of $25 \mathrm{c} . \mathrm{cm}$. of 1 in 400 carbolic acid in normal saline was given intrathecally under chloroform and ether anaesthesia. Morphine $1 / 6$ grain and atropine 1/100 grain were given four-hourly for the relief of spasms. During the next twenty-four hours there was very marked improvement. The spasms became less severe and occurred at half-hourly intervals. Improvement continued for four days, and then, as there was a slight relapse, another $\mathbf{8 0 , 0 0 0}$ units were given intravenously, and 120,000 intramuscularly. Ten days after admission there only remained some stiffness of the jaw and of the muscles of the spine and abdomen. $\mathrm{He}$ was discharged perfectly well at the end of four weeks. No renal symptoms were observed as a result of the carbolic injection. Cultures from the pus grew anaerobic organisms, but no actual $B$. tetani.

\section{CAse V}

Female, aged 19 years, domestic servant. Twelve days before admission she pricked her little finger with a pin on her dress. Five days before admission she complained of pain and stiffness in the neck, shoulders, and chest. The next day this was more marked, and she had difficulty in opening her mouth. These symptoms became worse until she was admitted. The first severe spasms of pain came on the night before she was admitted

State on Admission.-Risus sardonicus. Teeth could be separated between spasms. Extreme rigidity of the muscles of the neck, spine, chest, and abdomen. Profuse sweating. A small subcuticular whitlow of the little finger of the right hand marked the site of the original prick.

Severe spasms of the whole body occurred about every fifteen minutes, or on slight stimulation or movement of the limbs.

Treatment and Progress.-On admission 140,000 units were given intravenously, and 60,000 intramuscularly. On the next day another 100,000 were given intravenously. The whitlow was thoroughly treated surgically, and hydrogen peroxide dressings were applied.

The spasms became rapidly less severe, and after three days all reflex spasm had gone. The stiffness of the muscles of the jaw, spine, and abdomen graduaily relaxed. At the end of ten days she could raise her head from the pillow, and at the end of three weeks all spasms had passed off. She was discharged well at the end of five weeks.

\section{CASE VI}

Male, aged 73 years. Ten days before admission he trod on a nail, wounding his big toe, which went septic. On the day of admission he complained of stiffness of the jaw, so that he could not open his mouth properly. $\mathrm{He}$ also had pain in the back.

State on Admission.-Marked trismus and risus sardonicus. The teeth could be separated a distance of $1 / 2$ inch between spasms. There was rigidity of the neck muscles. Any manipulation of the limbs caused spasm of the muscles of the spine and limbs. Occasional slight spasms occurred, but these were not very severe.

Moderate arterio-sclerosis was present, but this was not exceptional for the age. On the right big toe there was a septic blister $1 / 2$ inch by 1 inch in size. This contained about $1 \mathrm{c.cm}$. of foul-smelling pus. The superficial raw surface was laid bare, and hydrogen peroxide dressings were applied. The culture of the pus from the wound yielded $B$. tetani and other anaerobes ; 40,000 units were given intrathecally, and 20,000 intravenously under chloroform anaesthesia. This was repeated the following day. The spasms, however, became more severe, and death took place two days after admission.

Report on the Pus.-Predominant organism: spore-bearing bacillus of anaerobic type ; a few characteristic of $B$. tetani. Cultures (anaerobic): Gram-positive sporebearing bacilli, some of which have the characteristics of $B$. tetani.

CASE VII

Male, aged 10 years. Seven days before admission he fell on to a barn floor and sustained a compound fracture of the right radius and ulna. No prophylactic antitoxin was given. Two days before admission he seemed unwell, and complained of pain in the back. Severe spasms began on the day of admission.

State on Admission.-Very severe spasms with extreme opisthotonos were almost continuous. Clenched teeth. Risus sardonicus.

Treatment and Progress.-In the seven hours before death 520,000 units were given-350,000 intravenously, and 170,000 intramuscularly, under chloroform and ether anaesthesia. There appeared to be some diminution in the severity of the spasms before death, which seemed to be due to prolonged spasm of the respiratory muscles causing respiratory failure. An extensive cellulitis involved the whole arm in the region of the fracture, and this could not be cleansed.

\section{CASE VIII}

Male, aged 12 years. Seven days before admission he fell down in the road and cut his left knee. The cut was three inches long and one inch deep, and dirty. It was stitched up, but no antitoxin was given. Eighteen hours before admission he complained of pain and stiffness in the left leg, which spread rapidly to the muscles of the back, neck and jaw.

State on Admission.--Very severe spasms every few minutes or on the slightest stimulation, with severe opisthotonos. Risus sardonicus present. The teeth could only be separated $1 / 4$ inch between the spasms. 
Treatment and Progress.-Under chloroform and ether anaesthesia 1,000 units were injected into the wound, 20,000 intramuscularly, 40,000 intrathecally, and 140,000 intravenously ; 160,000 were given during the next eight hours intramuscularly. The wound was thoroughly cleansed, and hydrogen peroxide dressings were applied four-hourly. Death took place twelve hours after admission, apparently from respiratory failure during a spasm. For a few hours before death the spasms were definitely less frequent and severe.

\section{CASE IX}

Male, aged $2 \frac{1}{2}$ years. Admitted with a history of four days' ear discharge and fits. Fourteen days before admission he sustained a superficial graze on the left elbow, which healed up, leaving no scar. Four days before admission he complained of bilateral earache, and one day later the right ear began to discharge. The day before admission he was not himself, and his mother noticed that he held his head on one side and could not open his mouth to eat. He had several attacks, described as a kind of fit, complained of pain in the back and legs, and made faces in which he "seemed to laugh."

State on Admission and Progress. - Teeth clenched. Masseters in severe spasm; risus sardonicus; abdomen board-like. Typical tetanic spasms occurred at first every hour, rapidly becoming more frequent and prolonged, so that for the few hours before death they were almost continuous. Sweating was profuse, and the rectal temperature rose to $110^{\circ}$ before death, which took place twenty-four hours after admission.

Treatment.-Intrathecally 30,000 units were given, and 20,000 intramuscularly. Chloral and bromide by the mouth, paraldehyde per rectum, and chloroform by inhalation for the spasms.

\section{CASE $\mathrm{X}$}

Male, aged 60 years, gardener. Ten days before admission he went to a doctor for a whitlow of the right thumb, which he had had for a few days. He was treated by hot fomentations. Two days before admission he complained of stiffness of the jaw, and the following day had a "sort of fit," in which his arms were drawn up.

State on Admission.-Risus sardonicus. Teeth could not be separated more than $1 / 4$ inch. The muscles of the neck, chest, spine, and abdomen were in a state of extreme rigidity, which was made worse by movement. Spasms of the arms, head, and back occurred every few minutes after admission. The patient was a bad subject, with some chronic bronchitis and emphysema, and marked general arterio-sclerosis.

Treatment and Progress. - The thumb-nail was removed, revealing some very foul-smelling pus from which anzerobes were grown; no $B$. tetani were identified. Hydrogen peroxide dressings were applied after the wound had been thoroughly cleaned. Under chloroform anaesthesia 120,000 units were given intramuscularly, and $30 \mathrm{c} . \mathrm{cm}$. of 1 in 400 carbolic in normal saline was given intrathecally. The following day the spasms were still very severe, and avertin was given per rectum. This relieved the spasms considerably, but death took place thirty-six hours after admission.

\begin{tabular}{|c|c|c|c|c|c|c|}
\hline \multicolumn{7}{|c|}{ Summary of Cases } \\
\hline No. & Age & S $3 x$ & $\begin{array}{c}\text { Incubation } \\
\text { Period. } \\
\text { Days }\end{array}$ & $\begin{array}{l}\text { Rate of } \\
\text { Onset. } \\
\text { Days }\end{array}$ & Dose A.T.S. & Result \\
\hline 1 & 11 & M & 11 & 3 & 234,000 & Rscovered \\
\hline 2 & 23 & M & $?$ & 8 & 240,000 & , \\
\hline 3 & 10 & M & 9 & 4 & 340,000 & , \\
\hline 4 & 11 & M & 16 & 5 & 360,000 & ," \\
\hline 5 & 19 & F & 7 & 5 & 303,000 & , \\
\hline 6 & 73 & M & 10 & 2 & 100,000 & Died \\
\hline 7 & 10 & M & 5 & 2 & 520,000 & ", \\
\hline 8 & 12 & M & 6 & 1 & 361,000 & " \\
\hline 9 & 3 & M & $?$ & 2 & 50,000 & " \\
\hline 10 & 60 & $\mathbf{M}$ & 10 & 2 & 120,000 & " \\
\hline
\end{tabular}

\section{The Wound}

The wounds in this series of cases were as follows: whitlow, two cases (both mild) ; acute otitis media ; compound fracture of radius and ulna; deep incised wound of leg; thorn in leg, with pus formation ; small splinter of wood below knee; septic blister of great toe; small abrasion of foot, with scab formation; no wound found.

The point worth noting in this series is the way in which superficial healing and scab formation over a trivial wound may create a cavity for anaerobes to grow in. This is well shown by Case VI, in which the wound, although started by a prick, became a superficial blister in which $B$. tetani could grow. Anaerobes were also grown from the pus sealed off by a scab in Case $I$, and by the thumb-nail in Case $x$. Cases viI and viII might have been prevented by the prophylactic use of antitoxin ; both these patient died.

\section{PRognosis}

These cases conform to the well-known fact that a long incubation period increases the chance of recovery. No case that lived in this series had an incubation period of less than seven days. Slow onset of symptoms is also a favourable sign. An index of the rate of onset is the length of time between the first symptoms of illness and the first severe generalized spasms. This period in all the five patients who lived was three days or more, whereas in the five who died it was two days or less.

Other factors which certainly make for an unfavourable issue are old age, and a very severe wound which cannot be thoroughly drained so that anaerobic organisms can continue to multiply and produce toxin after treatment has begun. With proper prophylactic use of antitoxin, however, these cases should never occur.

\section{Principles of Treatment}

The therapeutic value of antitoxin is still a disputed question. Toxin reaches the central nervous system via the motor nerve endings and the axis cylinders of the motor nerves. Once it has reached the central nervous system it becomes fixed to the nerve cells, and, with one exception, the experimental evidence shows that antitosin - -even in very large doses-has a negligible neutralizing effect upon it. The exception is an experiment by Sherrington. ${ }^{1}$ He injected monkeys in batches of twentyfive with $8 \mathrm{~m} .1 . d$. of pure toxin (no bacilli) into the outer head of the gastrocnemius muscle. From forty-seven to seventy-two hours later, when the early symptoms of tetanus had appeared, he injected 2,000 U.S.A. units of antitoxin per kilo, giving the serum by different routes in each batch of monkeys. His results showed that the intrathecal route was the most effective, with a percentage mortality of 44 , the intravenous next with 72 per cent., the intramuscular next with 88 per cent., and the subcutaneous least with 92 per cent. Until lately it has not been possible to give a corresponding dose of antitoxin to man, because a sufficiently high titre antitoxin has not been available. This may be one of the reasons why the therapeutic use of antitoxin fell into disrepute. Now, however, that more concentrated antitoxin has been prepared, it has become possible to give doses to man corresponding to those used by Sherrington in monkeys: This has been done by A. E. Paterson ${ }^{2}$ of Sydney, who, using doses of this magnitude, records nineteen recoveries in a series of twenty-six consecutive cases in children. Owing to the difficulty of giving sufficient antitoxin by the intrathecal route this was not used, the bulk being given by the intravenous route, and the rest intramuscularly. In four of Paterson's cases that recovered the incubation period was only seven days and in one only six, so that this very high recovery rate cannot be 
attributed to the mildness of the infection. Manson, ${ }^{3}$ in America, agrees with Paterson's views, and emphasizes the importance of early administration of large doses of antitoxin by the intravenous route.

Topley and Wilson ${ }^{4}$ suggest that the difference between Sherrington's results and the therapeutic results obtained in man may be due to the fact that he used toxin only and not bacilli. In man the effects obtained with antitoxin would appear to depend, not only on whether it can neutralize toxin which has reached the nervous system, but on the neutralization of toxin in the wound before it has become attached. The apparent success of very large doses of antitoxin may perhaps be explained by the neutralization of toxin in the tissues before it has reached the nervous system. In this way large doses of antitoxin, combined with local treatment of the wound, may prevent further absorption of toxin. If this can be done before a lethal dose has reached the nervous system, death may be prevented.

Treatment of active tetanus may therefore be directed along the following lines. It may aim at preventing further production of toxin in the wound by thorough local measures; at neutralizing any further toxin which is formed in the wound in spite of local treatment by giving large doses of antitoxin; at neutralizing toxin which is already fixed to the nervous system by giving large doses of antitoxin (assuming that this is possible from Sherrington's experiments) ; at neutralizing toxin by nonspecific measures, such as the intrathecal injection of carbolic acid, as used by Suvansa ${ }^{5}$; at preventing spasms and pain ; and at maintaining strength by general measures.

In a small series of clinical cases such as this it is impossible to say how far antitoxin was of use; still less is it possible to compare the value of the routes used. Ten cases were treated and five recovered. This does not compare with Paterson's results, but it should be remembered that of the five patients who died two were men of 60 and 73, while two others had deep infected wounds of great severity, which prophylactic antitoxin might have saved. The five patients who recovered had doses of antitoxin ranging from 234,000 to 360,000 units, given chiefly by the intravenous and intrathecal routes. In any case of tetanus it is extremely difficult to give a prognosis, and therefore to assess the results of treatment. Large doses of antitoxin, however, did appear to influence the course of the disease favourably in these cases, by lessening the severity and frequency of the spasms. The only disadvantage of such treatment is the expense. The cost of 100,000 units of serum is approximately $£ 4$.

\section{Summary}

Tetanus is still sufficiently common in some districts to make it worth while re-emphasizing the importance of prophylactic antitoxin, even after trivial wounds.

When the disease has developed the following rules should be observed.

1. Diagnosis should be made and treatment begun as early as possible. Pain and stiffness in the back of the neck and spine may be the earliest symptom. These, associated with slight stiffness of the jaw and rigidity of the abdomen, should suggest tetanus.

2. Local treatment of the wound should be thorough, and should be done at the earliest possible moment.

3. Antitoxin should be given as soon as possible in very large doses, preferably by the intravenous route.

4. Every effort must be made by symptomatic measures to reduce spasms and allay pain. For this purpose avertin may prove of value
5. The strength must be maintained by giving plenty of nourishment. For this to be achieved good narsing is essential. Glucose should be given freely.

I am indebted to Dr. J. A. Wright and Dr. G. S. Haynes for permission to observe and record Cases II and III, and to Dr. C. H. Whittle for the bacteriological examinations.

\section{REFERENCES}

${ }^{1}$ Sherrington, C. S.: Lancet, 1917, ii, 964

2 Paterson, A. E:: Med. Journ. of Australia, 1930, i, 839.

3 Manson, M. H.: Minnesola Med., 1931, i, 142.

4 Topley, W. W. C., and Wilson, G. S.: The Principles of Bacteriology and Immunity, Loadon, 1929, ii, 1158.

s. Suvansa, S.: Lancet, 1931, i, 1075.

\section{ENDOCRINE THERAPY IN DYSMENORRHOEA}

\section{BY}

WALTER P. KENNEDY, B.Sc., Ph.D., L.R.C.P. \& S. BEIT MEMORIAL RESEARCH FELLOW

(From Dr. James Young's Gynaecological Clinic, Royal Infirmary, and Physiology Department, Edinburgh University.)

The common complaint of dysmenorrhoea has caused a widespread controversy as to its aetiology and treatment. Many types of the disorder have been described in terms which have sometimes tended to confuse the problem. This is exemplified by the presupposition of a condition such as "obstruction" "without proof of its existence, or by emphasizing the "congestive" element which is normally present in the premenstrual and menstrual phases. Just as a distinction has been drawn between hyperpiesis and hyperpiesia, we prefer to speak of dysmenorrhoea as a symptom accompanying some recognized primary condition such as cervicitis or salpingitis, and dysmenorrhoea as an entity in the absence of any detectable disease. For the symptom dysmenorrhoea, the term " menorralgia " has been suggested, and if it could gain wide acceptance it would have much to commend it. Our special concern here is with the severe cases of dysmenorrhoea, sometimes called primary, idiopathic, essential, or intrinsic, which seek hospital treatment. In these the simpler remedies such as mild analgesics, attention to the bowels, and such exercises as those described by Dr. A. Sanderson Clow, must be frequeritly amplified by more intensive treatment. This falls into two groups: operative procedures and endocrineltherapy. Examination of these seemed desirable with a view to throwing light on the nature of that disordered physiological function which becomes prominent as pain, and affording a rationale for ovarian therapy. This preliminary study was therefore undertaken, and forms part of a more extensive investigation on menstrual physiology and pathology.

The clinical picture presented by the patients who come to hospital for relief is of the following kind. They are generally unmarried and nulliparous, and the onset of the complaint usually dates from two to three years after the menarche, though in a few cases it coincides with the first menstruation. Apart from the critical time the patient feels well, but some days or hours before the onset of each period, strong cramp-like or colicky pains commence in the mid-line of the lower abdomen, and less frequently in the low lumbar or sacral regions. The pain reaches its greatest intensity at or just after the beginning of the flow, and then commonly subsides. It may, however, occasionally continue throughout, and even for one or two days after the period. The pain is often so severe as to necessitate the patient going to bed, and is accompanied by greater or lesser degrees of malaise, anorexia, vomiting, headache, and sometimes manifestations of reduced vasomotor control as evinced by cold hands and feet, etc. The periodicity may be either regular or slightly 